\title{
Comparison between semi-automated segmentation and manual point-counting methods for quantitative analysis of histological sections
}

\author{
José M. Amenábar, Gabriela B. Martins, Karen Cherubini and Maria A. Z. Figueiredo \\ Department of Oral Medicine, Faculty of Odontology, Pontifícia Universidade Católica do \\ Rio Grande do Sul, Rio Grande do Sul, Brazil
}

(Received 20 March and accepted 26 July 2006)

\begin{abstract}
The aim of the present study was to compare results obtained using the manual pointcounting method with results obtained using the semiautomated segmentation method, in the quantitative evaluation of histological sections by light microscopy. The tongues of 40 rats (Ratus norvergicus) were processed using the paraffin technique, in which a 5$\boldsymbol{\mu}$ m-thick tissue slice cut from each tongue is stained with picrosirius and methyl green. Three digital images were captured randomly from each section, which were later converted to black-and-white. The collagen fibers shown in each image were quantified using the semi-automated segmentation method and the manual point-counting method. The results obtained using the 2 different quantification methods were compared using the paired $t$-test and Pearson's correlation test, with statistical analysis using the methods of Bland and Altman. The $t$-test indicated that there was no significant difference in results between the 2 different quantification methods. Pearson's correlation test indicated a strong correlation $(r=0.987)$, and a comparison of the methods using the Bland and Altman plot indicated no significant error. These findings indicate that both quantification methods can be reliably used to evaluate tissue sections, without discrepancies between the 2 methods. (J. Oral Sci. 48, 139-143, 2006)
\end{abstract}

Keywords: image analysis; histology; morphometry.

Correspondence to Dr. José Miguel Amenábar, Hospital São Lucas PUC/RS, Av. Ipiranga, 6690 - Sala 231 - $2^{\circ}$ andar, CEP: 90610-000, Porto Alegre-RS, Brazil

Tel: +55-51-3320-3254

Fax: +55-51-3320-3254

E-mail: jamenaba@terra.com

\section{Introduction}

Evaluation of the physiological or pathological condition of tissues, cells or cellular organelles requires quantitative methods of analysis (1-4). According to Bolender (5), the development of such methods has been one of the principal advances in experimental biology in the last few years.

Image analysis can depend on variables such as shape, orientation, area and volume (6). The measurement of area of microscopic objects can be performed directly by light microscopy, on projected images using a microprojector, or on digitally captured images (7).

Among the methods available for the quantification of area in histological sections is planimetry. Conventional methods of planimetry include linear integration, counting of points and the use of a polar planimeter (7). Counting of points is a classical planimetric method for analysis of tissue sections. Counting of points requires a test system or grid, which is composed of an assemblage of points whose arrangement can be regular or random, and which is superimposed on the image (8). The grid permits the determination of the proportion of the volume or area of a histological section that is taken up by a given structure, by counting the points that touch the structure (9).

Advances in informatics have led to improvements in analysis of histological sections, via improvements in classical techniques and development of new methods of image analysis. Tissue sections have a spatial component (morphological appearance), and can exhibit hundreds of different colors depending on the staining method used. In digitization of a histological section, a computer produces an image composed of pixels (10). In this image, each pixel has a specific color that can be used for analysis. In semiautomated methods using segmentation, it is possible to 
distinguish between different particles and to distinguish between particles and the background. This distinction allows the program to recognize contiguous pixels and to group them in regions. There is no formal model for segmentation, which is an empirical process based on analysis of the histogram of the image. Each image is converted into binary images (8-bit), with an intensity scale of 256 shades of gray, ranging from 0 (black) to 255 (white). To assure that the illumination of the microscope does not affect relative image intensities, it should be kept constant. Image analysis requires selection of a cut-off point for segmentation in the histogram.

The aim of the present study was to compare results obtained using the manual point-counting method with results obtained using the semi-automated segmentation method in the quantitative evaluation of histological sections by light microscopy.

\section{Materials and Methods}

Forty tongues from rats (Ratus norvergicus) were processed using the paraffin technique, in which $5-\mu \mathrm{m}$-thick tissue sections were obtained from each tongue and stained with picrosirius and methyl green. This staining method stains collagen fibers red, while the other histological components are counterstained green. Three images were captured randomly from each section, using a light microscope (Olympus AX 70, Tokyo, Japan) with a 20× objective (Olympus U-PMTVC, Tokyo, Japan), coupled to a digital image-capture system.

The captured images were analyzed using the software Image-Pro Plus version 4.0 (CyberMedia, NY, USA). The tonalities used in the analysis were in the red range, which is the color of the stained collagen fibers. Consequently,

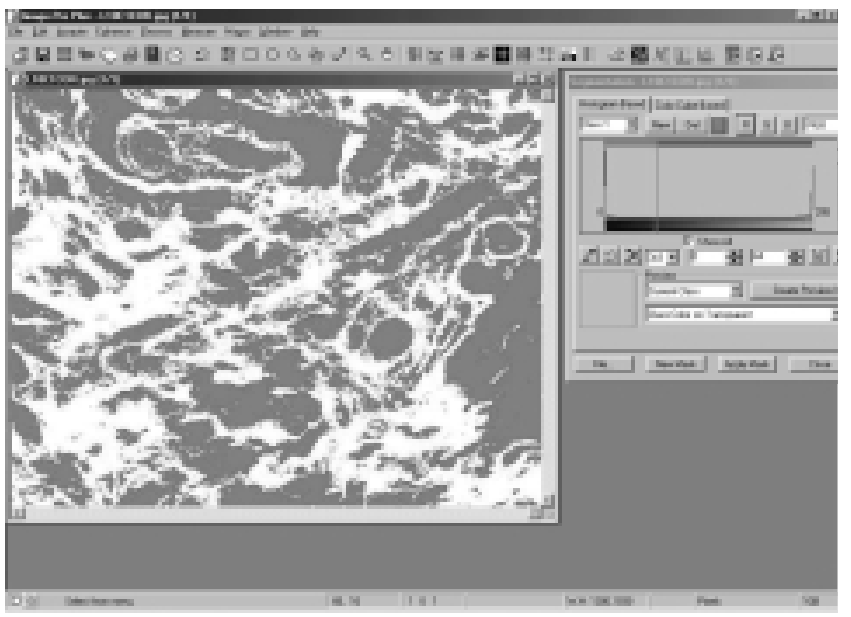

Fig. 1 Mask applied in the software to distinguish the collagen fibers from other tissue structures, with consequent conversion of the image to black-and-white. a red-colored mask was applied to the images to convert them to black-and-white (Fig. 1). In each new image thus created, the area occupied by collagen fibers was white and the rest of the tissue was black (Fig. 2). Finally, erosion and dilatation filters were applied to the images to improve them.

After the above treatment, each image was analyzed using the semi-automated segmentation method and the manual point counting method.

\section{Semi-Automated Segmentation Method}

A square was drawn for each image, with an area of $39,034 \mu \mathrm{m}^{2}$, which is the field area of a $20 \times$ objective. For each image, the white area (corresponding to the collagen fibers) enclosed by the square was measured in $\mu \mathrm{m}^{2}$ (Fig.

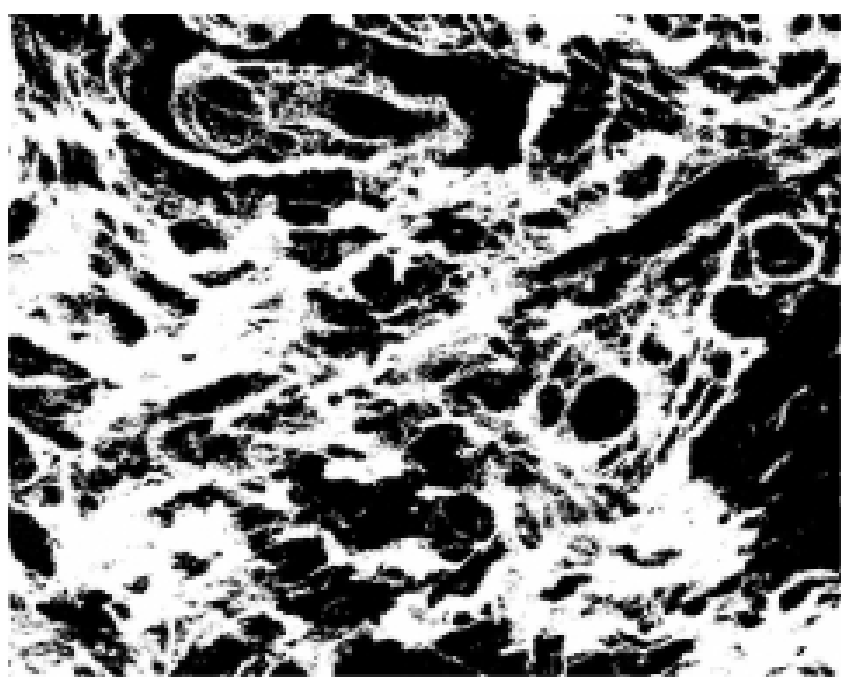

Fig. 2 New image created: collagen fibers are represented by white areas, and the rest of the tissue is black.

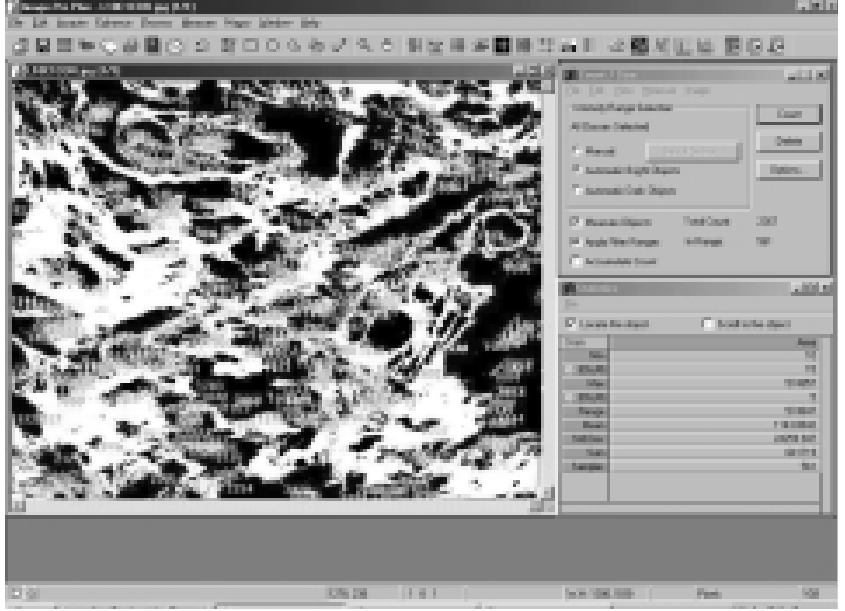

Fig. 3 Image analysis using the semi-automated segmentation method. The program calculates the areas occupied by the white color. 
3 ), and the mean of this area was determined for each tissue section (mean of 3 images). The percentage of the total area of each section that was taken up by the white area (area occupied by collagen fibers) was calculated using the following formula: area occupied by fibers (mean of 3 images) $\times 100 /$ total area.

\section{Manual Point-Counting Method}

In accordance with the protocol proposed by Amenábar et al. (8), a 320-point grid was overlayed on each image. For each image, the points touching the white area corresponding to collagen fibers were counted (Fig. 4), and the mean of this point count was determined for each tissue section (mean of 3 images). The percentage of the total area of each section that was taken up by the white area (area occupied by collagen fibers) was calculated using the following formula: number of points touching the white area (mean of 3 images) $\times 100 /$ number of total points.

\section{Statistical Analysis}

The comparison of results between the 2 methods was performed using the paired $t$-test, with the level of significance set at 5\%. Pearson's correlation test was used

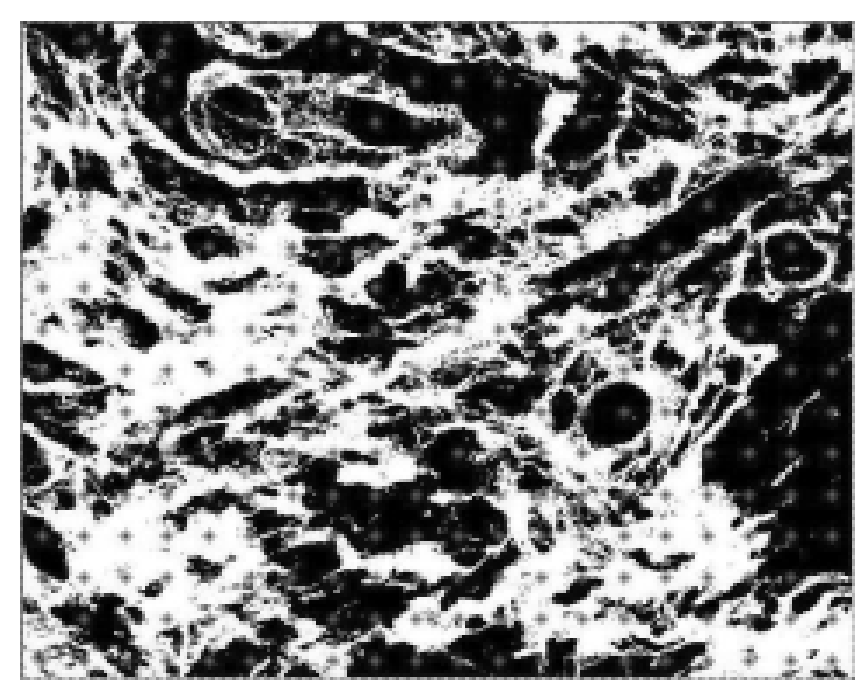

Fig. 4 Grid overlayed on the image, counting the points touching white areas corresponding to collagen fibers. to determine the correlation between the results of the 2 methods. The statistical analysis proposed by Bland and Altman (11) was used to establish the degree of concordance between the 2 methods.

\section{Results}

Table 1 shows the values for the relative frequency of collagen fibers obtained using the semi-automated segmentation method and manual point-counting method. The $t$-test for paired samples indicated that there was no significant difference between the results obtained with the 2 quantification methods $(P=0.339$, Table 1$)$.

Pearson's correlation test indicated a strong correlation ( $\mathrm{r}=0.987, P=0.000)$. Comparison between the 2 quantification methods using the Bland and Altman plot (11) indicated that there was no significant error. Fig. 5 shows the difference between the results of the 2

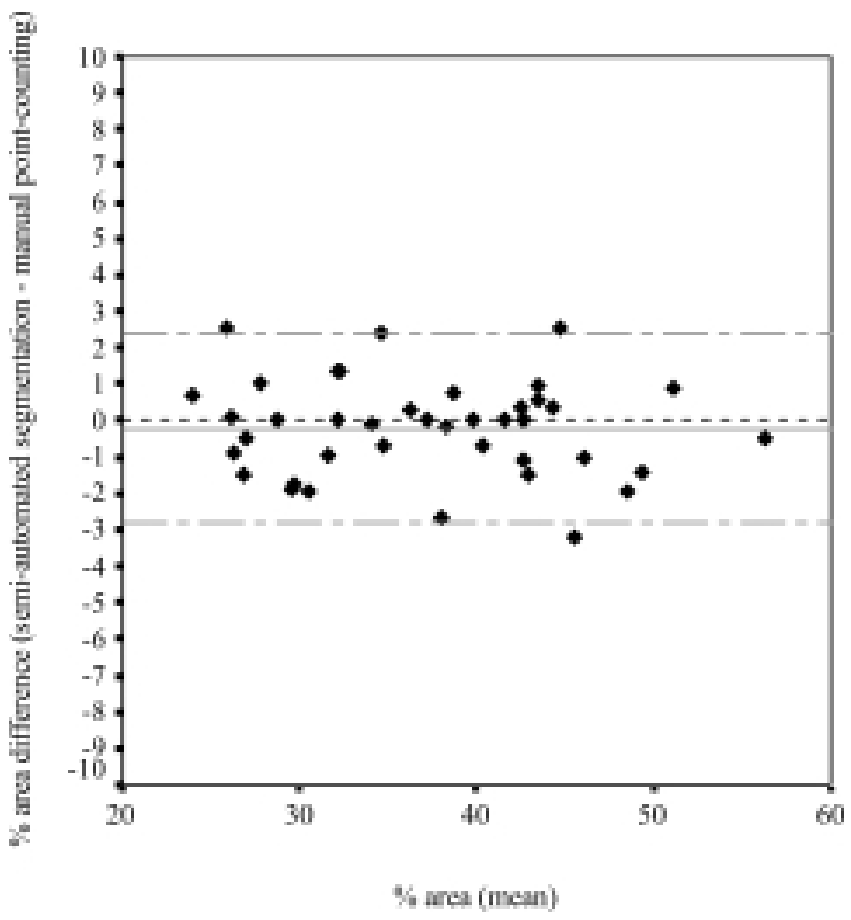

Fig. 5 Difference between the results for the semi-automated segmentation method and manual point-counting method, versus their respective means, showing the error and limits of agreement.

Table 1 Relative frequency of collagen fibers obtained using the semi-automated segmentation method and manual pointcounting method, Porto Alegre, 2005

\begin{tabular}{lccc}
\hline \multicolumn{1}{c}{ Technique } & $\begin{array}{c}\text { Collagen fibers (\%) } \\
\text { Mean and standard deviation }\end{array}$ & $\begin{array}{c}\text { Other histological components (\%) } \\
\text { Mean and standard deviation }\end{array}$ & $\boldsymbol{p}^{*}$ \\
\hline Semi-automated segmentation & $37.09 \pm 8.03$ & $62.91 \pm 8.03$ & 0.339 \\
Manual point-counting & $37.29 \pm 8.18$ & $62.71 \pm 8.18$ & \\
\hline
\end{tabular}

${ }^{*} t$-test for paired samples. Significantly different if $P \leq 0.05$. 
quantification methods versus their respective mean. The difference between the means of the 2 quantification methods was $0.2 \%$. Fig. 5 also shows the estimated error for the 2 quantification methods, and the upper and lower limits of concordance for a $95 \%$ confidence interval. The data in Fig. 5 shows that the majority of the values obtained using the 2 quantification methods are within the confidence interval.

\section{Discussion}

Several statistical approaches have been proposed to determine the concordance between 2 methods of quantitative evaluation, including correlation coefficients, $t$-test and the Bland and Altman method. Correlation coefficients measure the strength of a relationship between 2 variables, while significance tests such as the $t$-test determine whether there is a significant difference between 2 values or the means of 2 groups of values (12). The method of Bland and Altman (11), on the other hand, determines the mean and standard deviation of differences between values obtained using different quantification methods, and also plots the values obtained versus the difference between the means of the 2 quantification methods. There is controversy as to which is the better statistical method for determining concordance between distinct quantification methods. Therefore, in the present study, all 3 statistical approaches were used.

In the Bland and Altman method, the interpretation of the results depends on the difference between the values obtained using the 2 quantification methods. If this difference is not of sufficient magnitude to cause problems with interpretation, the difference between the 2 sets of values is not considered significant $(13,14)$. This means that if the results of the repeated measurements were constant (i.e., if the differences between the obtained values were small), such as in the present study $(0.2 \%)$, the results of either of the 2 quantification methods would not be significantly affected by random error (13).

In the present study, for 19 of the 40 tongues sampled, the means of the differences between the 2 quantification methods were close to zero. This indicates that there was strong concordance between the 2 quantification methods (15).

The interpretation of the results depends on the magnitude of the variables that are being analyzed (14). Consequently, because the semi-automated segmentation method and manual point-counting method produced similar results, these 2 methods can be considered to have good concordance. This indicates that they are reliable alternative methods for the quantitative analysis of histological sections.
Pearson's correlation test revealed a strong correlation between the 2 present quantification methods $(r=0.987)$. However, because investigators generally try to consider all possible variations for a set of data when they compare the behavior of 2 quantification methods or their results, one can expect a strong correlation when quantitative methods that have the same purpose are compared (12). Statistical analysis using the $t$-test for paired samples showed that there was no significant difference between the results obtained with the 2 present quantification methods.

Although the present statistical analysis showed no significant difference between the results obtained by the semi-automated segmentation method and manual pointcounting method, these 2 methods are not interchangeable. The manual point-counting method allows analysis of images visualized directly under the microscope (as well as digitized images), in cases where a computer or appropriate software is not available. However, the manual point-counting method requires a considerable number of grid points to be reliable (9); i.e., to provide a minimum level of confidence of $95 \%$ (8).

On the other hand, the semi-automated segmentation method requires the use of software to segment the image. In addition, care must be taken with the illumination and focus of the microscope in image capturing. Illumination should be constant for all images, because even small variations can interfere with the perception of the camera and alter the results (16).

In the present study, we used the picrosirius and methyl green staining method. Picrosirius stains collagen fibers, and methyl green stains the background. This staining method was used because of its convenience in distinguishing the structures analyzed. Both the semiautomated segmentation method and the manual pointcounting method can also be used with many other histological stains for quantification of distinct tissue structures.

The present results suggest that both the manual pointcounting method and semi-automated segmentation method can be reliably used for evaluation of histological sections without discrepancies between the results of the 2 methods.

\section{References}

1. Weibel ER (1989) Measuring through the microscope: development and evolution of stereological methods. J Microsc 155, 393-403

2. Mayhew TM (1992) A review of recent advances in stereology for quantifying neural structure. $\mathrm{J}$ Neurocytol 21, 313-328

3. Roberts N, Puddephat MJ, McNulty V (2000) The 
benefit of stereology for quantitative radiology. $\mathrm{Br}$ J Radiol 73, 679-697

4. Andersen BB, Pakkenberg B (2003) Stereological quantitation in cerebella from people with schizophrenia. Br J Psychiatry 182, 354-361

5. Bolender RP. (1992) Biological stereology: history, present state, future directions. Microsc Res Tech 21, 255-261

6. Hamilton PW, Allen DC (1995) Quantitative clinical pathology. Blackwell Science, Oxford, 11-22

7. Manderim-de-lacerda C (1995) Quantitatives methods in morphology. EDUERJ, Rio de Janeiro, 60-69 (in Portuguese)

8. Amenábar JM, Hugo FN, Fossati AMC, Padilha DMP (2003) The use of stereology as a method in histological research. Rev Fac Odontol Porto Alegre 44, 62-65 (in Portuguese)

9. Dickson JM, Weavers HM, Mitchell N, Winter EM, Wilkinson ID, Vanbeek EJ, Griffiths PD (2003) Choice of cross size in stereology - a cautionary note. Neuroradiology 45, 896-899
10. Castleman KR (1996) Digital image processing. Prentice-Hall, New Jersey, 15-72

11. Bland JM, Altman DG (1986) Statistical methods for assessing agreement between two methods of clinical measurement. Lancet 1, 307-310

12. Bland JM, Altman DG (1996) Measurement error proportional to the mean. BMJ 313, 106

13. Altman DG (1991) Practical statistics for medical research. Chapman \& Hall, London, 55-59

14. Bland JM, Altman DG (1995) Comparing methods of measurement: why plotting difference against standard method is misleading. Lancet 346, 10851087

15. Hulley SB, Cummings SR, Browner WS, Grady D, Hearst N, Newman TB (2001) Designing clinical research: an epidemiologic approach. 2nd ed, Lippincott Williams \& Wilkins, Baltimore, 37-50

16. Sims AJ, Bennett MK, Murray A (2002) Comparison of semi-automated image analysis and manual methods for tissue quantification in pancreatic carcinoma. Phys Med Biol 47, 1255-1266 